\title{
Gender differences in patients with COVID-19: a narrative review
}

\author{
Immacolata Ambrosino1, Elena Barbagelata ${ }^{2}$, Elena Ortona ${ }^{3}$, Anna Ruggieri ${ }^{3}$, Grace Massiah ${ }^{4}$, \\ Orazio Valerio Giannico ${ }^{5}$, Cecilia Politi ${ }^{6}$, Anna Maria Moretti ${ }^{7}$
}

${ }^{1}$ Local Healthcare Unit of Bari, Health District 13, Bari; ${ }^{2}$ Department of Internal Medicine, Lavagna Hospital, Genoa; ${ }^{3}$ Center for Gender Specific Medicine, Istituto Superiore di Sanità, Rome; ${ }^{4}$ Plastic and Reconstructive Surgery, IRCCS Giovanni Paolo II, Bari; ${ }^{5}$ Department of Prevention, Local Health Authority of Taranto; ${ }^{6}$ Head of Gender Medicine Area F.A.D.O.I. (Federazione delle Associazioni dei Dirigenti Ospedalieri Internisti), Isernia; ${ }^{7}$ President of GISeG, Italian Group for Health and Gender, Bari, Italy

\begin{abstract}
In December 2019 a novel coronavirus emerged in Wuhan, China causing many cases of severe pneumonia. World Health Organization (WHO) named this disease Coronavirus Disease 2019 (COVID-19). The infection has rapidly spread across China to many other countries, and on March 12, 2020 the WHO declared pandemic outbreak of COVID-19. As of May 16, 2020, COVID-19 has been diagnosed in more than 4,490,000 patients, associated to 305,976 deaths worldwide; in Italy 224,760 COVID19 cases have been reported with 31,763 deaths. The main routes of transmission are respiratory droplets and direct contact with infected people, so numerous prevention strategies are employed to mitigate the spread of disease, including social distancing and isolation. The aim of this narrative review is to underline gender differences in epidemiology, etiopathogenesis, risk factors, clinical presentation, diagnosis, prognosis and mortality of patients infected with SARS-CoV-2. Currently data on the sex indicators
\end{abstract}

Correspondence: Anna Maria Moretti, President of GISeG (Bari), via Napoli 215, 70123 Bari, Italy.

E-mail: moretti.am@libero.it

Key words: Gender differences; COVID-19.

Authors' contributions: IA, study conception and design, revision of all abstracts, manuscript drafting; EB, revision of all abstracts, critical revision; OVG, EO, AR, GM, CP, critical revision; AMM, study conception and design, critical revision. All the authors have read and approved the final version of the manuscript and agreed to be accountable for all aspects of the work.

Conflict of interests: The authors declare no conflict of interest

Received for publication: 18 May 2020.

Accepted for publication: 19 May 2020.

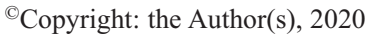

Licensee PAGEPress, Italy

Monaldi Archives for Chest Disease 2020; 90:1389

doi: 10.4081/monaldi.2020.1389

This article is distributed under the terms of the Creative Commons Attribution Noncommercial License (by-nc 4.0) which permits any noncommercial use, distribution, and reproduction in any medium, provided the original author(s) and source are credited. for admitted or deceased patients are only available, but there is no analysis about other gender indicators. The data considered in our study are the only currently available in the literature, but it is appropriate to implement a specific analysis with all gender indicators to identify appropriate strategies. Moreover, the evaluation of a health service efficiency is a key element to define gender outcomes. Knowing the gender differences in COVID-19 outbreak would be a fundamental tool to understand the effects of a health emergency on individuals and communities as well as to carry out effective and equitable policies, public health measures and targeted solutions.

\section{Introduction}

In December 2019, a series of pneumonia cases caused by a novel coronavirus (later referred to as SARS-CoV-2) emerged in Wuhan, China. World Health Organization (WHO) named this disease as Coronavirus Disease-2019 (COVID-19) on February 11,2020 . The infection has rapidly spread across China to many other countries, and on March 12, 2020 the WHO declared pandemic the COVID-19 outbreak [1]. As of May 16, 2020, COVID19 has been diagnosed in more than 4,490,000 patients and associated with 305,976 deaths [2] worldwide; in Italy 224,760 cases with 31,763 deaths [3] have been reported.

The main routes of transmission are respiratory droplets and direct contact with infected people. The COVID-19 virus spreads through droplets from mouth or nose when an infected person coughs or sneezes. Governments of many countries implemented several restrictive measures to contain the spread of the infection, including careful infection control, contact tracing, social distancing and isolation.

It has been estimated that the median incubation period is 5.1 days and that $97.5 \%$ of the infected subjects will develop symptoms within 11.5 days of infection. Patients infected with SARSCoV-2 can present a wide range of symptoms ranging from mild to severe. Fever, cough, and shortness of breath are the most common symptoms [4]. It has also been reported that about $2-10 \%$ of the patients with COVID-19 had gastrointestinal symptoms such as vomiting, diarrhea, and abdominal pain [4]; diarrhea and nausea preceded the development of fever and respiratory symptoms in $10 \%$ of patients [4]. Other symptoms were hyposmia or anosmia, gustatory dysfunction, and conjunctivitis.

The RT-PCR detection of viral nucleic acid test on samples from oro/rino-pharyngeal swab test was the most reliable established laboratory diagnosis method for SARS-CoV-2. 
To date, there are no specific vaccines nor treatments available for COVID-19. Several studies are ongoing to evaluate effective therapeutic strategies and specific protocols.

The aim of this narrative review is to underline gender differences in epidemiology, etiopathogenesis, clinical presentation and mortality of patients infected with novel coronavirus. Recognizing gender differences in COVID-19 is a fundamental tool in understanding the primary (health protection) and secondary (social, economic) effects of this health emergency on individuals and communities, and creating effective and equitable policies as well as targeted solutions.

\section{Methods}

Studies were identified through Pubmed systematic research until May 7, 2020. The search keywords were "SARS-CoV 2", "COVID-19", "gender", "estrogen", "androgen" and "coronavirus". The studies were selected and reviewed for potential inclusion. Studies written in languages other than English were excluded. Two authors (IA and EB) reviewed all study abstracts. Studies were included if they analyzed gender differences in SARS-COV-2 infection. All selected studies are qualitatively analyzed.

\section{Epidemiology}

Many studies underlined that in China men were more infected by COVID-19 (51\%) [5] than women, most patients were aged 3079 years [6] and the elderly with comorbidities had more severe conditions [7].

The study of Huang et al. [8] on 41 inpatients with laboratoryconfirmed COVID-19 infection, showed that most of the infected patients were men $(73 \%)$, the median age of infected patients was 49.0 years (IQR 41.0-58.0) and less than half of infected men had underlying diseases $(32 \%)$, including diabetes $(20 \%)$, hypertension $(15 \%)$, and cardiovascular disease $(15 \%)$.

Wang's study [7] enrolled 69 patients with confirmed SARSCoV-2, of whom 32 patients (46\%) were men, and 37 patients (54\%) were women, the median age of the patients was 42.0 years and the elderly and the patients with underlying comorbidities developed severe conditions. Chen conducted a retrospective, single-centre study [9] and observed a greater number of men than women in the 99 cases of $2019-\mathrm{nCoV}$ infection, in fact $32 \%$ were female and $68 \%$ were male with a mean age of $55 \cdot 5$ years. None of the 99 patients were medical staff. About half of patients (51\%) had chronic diseases, mainly cardiovascular and cerebrovascular diseases and diabetes. Wang [4] performed a retrospective study which included 138 hospitalized patients with confirmed COVID19 of whom $45.7 \%$ were women and $54.3 \%$ were men; the median age of inpatients was 56 years (IQR, 42-68; range, 22-92 years). The retrospective study by Zhou et al. [10] included 191 inpatients with COVID-19 with a median age of $56 \cdot 0$ years (IQR 46.0$67.0)$, ranging from 18 years to 87 years; 72 (38\%) were females and $119(62 \%)$ were males. The meta-analysis conducted by $\mathrm{Li}$ [11] confirmed these studies, in fact showed that males took a larger percentage in the sex distribution of COVID-19 patients $(60 \%)$. In a study [12] of 1099 patients with COVID-19 from 552 hospitals in 30 provinces in China, $58 \%$ of the patients were men and $41.9 \%$ of the patients were female and the median age of the patients was 47 years. Only one study of 140 patients with
COVID-19 in China [13] found an equal distribution by sex, in fact an approximately $1: 1$ ratio of male $(50.7 \%)$ and female $(49.3 \%)$ COVID-19 patients was found, with an overall median age of 57.0 years and the majority $(70 \%)$ of them were more than 50 years old.

The Report of the WHO-China Joint Mission on COVID-19 [14] as of $20^{\text {th }}$ February 2020, showed that the median age was 51 years with the majority of cases (77.8\%) aged between 30-69 years; among reported cases, $51.1 \%$ were male, $77.0 \%$ were from Hubei and $21.6 \%$ were farmers or laborers by occupation.

The Korean Report [15] showed that in total confirmed cases, two peaks of age groups were 20 years and 50 years; among the confirmed cases in Gyeonggi-do where the third-highest number of patients was observed, the peak age group was 30 years. Moreover, only $37.7 \%$ were male in total confirmed cases, but among the confirmed cases in Gyeonggi-do, 59.1\% were male. The distribution may reflect the movement and the social activities of individuals in different societies. The female prevalence in Korean COVID-19 patients of 20 years old is probably due to the outbreak in a religious group, whose demographic composition includes women mostly.

In Italy, first data reported in Lombardy Region reported a major prevalence of COVID-19 pneumonia in men and older patients. The retrospective observational study conducted by Grasselli [16] showed that males were 82\% (1304 of 1591 patients), while females were $18 \%$ (287 of 1591 patients). The median age was 63 years. In particular, in the age groups 51-60, $61-70$ and $71-80$, the majority of patients were men $(83 \%, 81 \%$ and $82 \%$, respectively) versus women $(17 \%, 19 \%$ and $18 \%$, respectively). Patients in these age groups had more comorbidities, in particular hypertension was the most common comorbidity, followed by cardiovascular disease and hypercholesterolemia. In a review conducted by Lovato [17], 57.5\% of a total of 1556 hospitalized patients with COVID-19 were males and the mean age was 49.1 years. According to the ISTAT-ISS report published on May $4^{\text {th }}$ 2020 [18], 52.7\% of the COVID-19 cases were female and the median age was 62 years. In the age groups 0-9 years, 60-69 and 70-79 years, a greater number of cases of males was observed. In the age group $>90$ years, the number of females was more than triple compared to males probably due to the clear prevalence of women in this age group.

Also interesting are data about medical staff: from $7^{\text {th }}$ January to $11^{\text {th }}$ February 2020 Chu [19] conducted a retrospective study among all 54 hospitalized medical staff from Tongji Hospital diagnosed with COVID-19. Most of those cases were from other clinical departments (39 cases - 72.2\%) rather than emergency department (2 cases $-3.7 \%$ ) or medical technology departments (10 cases $-18.5 \%$ ). There were categorized as common-type 11 out of 54 patients with COVID-19, while 40 as severe-type, and 3 as critical-type. Among 11 common-type patients, 5 cases $(45.5 \%)$ were females and 6 cases $(54.5 \%)$ were males. Unexpectedly, the median age for the commontype patients was significantly older than severe/critical-type patients ( 47 years vs 38 years; $\mathrm{p}=0.015$ ). Such differences may be explained by the lower ratio of elderly people among the medical staff and by the longer work time and higher work intensity of the medical staff aged 38 years or so. More male patients (30/43, 69.8\%) were found with severe/critical-type as compared with females $(13 / 43,30.2 \%)$. All 54 patients conducted SARS-Cov-2 nucleic acid tests, but only 38 were positive for the tests. The 16 patients negative for SARS-Cov-2 tests, showing typical pathological changes in CTscans were however diagnosed as COVID-19. The median age of patients positive (39 years) for SARS-Cov-2 tests was comparable to patients negative (46 years) to the tests. In both groups higher pro- 
portion of male patients were observed, in positive for SARS-Cov-2 tests: $24 \mathrm{M}(63.2 \%)$ and $14 \mathrm{~F}(36.8 \%)$, in negative for SARS-Cov-2 tests $12 \mathrm{M}(75 \%)$ and $4 \mathrm{~F}(25 \%)$.

\section{Physiopathology}

Full-genome sequencing and phylogenic analysis indicated that the coronavirus that causes COVID-19 is a beta-coronavirus in the same subgenus as the severe acute respiratory syndrome (SARS) virus (as well as several bat coronaviruses), but in a different clade. The structure of the receptor-binding gene region is very similar to that of the SARS coronavirus, and the virus has been shown to use the same receptor, the angiotensin-converting enzyme 2 (ACE2), for cell entry [20,21].

ACE2 is widely distributed in the heart, kidney, lung, and testis and may show differences in different ages and sexes. SARS-CoV2 invades human alveolar epithelial cells through ACE2 [22]. Interestingly, one study [23] found that the expression of ACE2 was more elevated in Asian men, which might be the reason for the higher prevalence of COVID-19 in this subgroup of patients than in women and patients of other ethnicities. ACE2 has been shown to play a protective role in acute respiratory distress syndrome. In fact, upon ACE2 down-regulation, mice showed more severe lung failure [21]. A study [24] in animal models underlined that ACE2 expression is dramatically reduced with aging in both genders: young-adult $v$ s old $\mathrm{p}<0.001$ (by $78 \%$ in male and $67 \%$ in female, respectively) and middle-aged $v s$ old $\mathrm{p}<0.001$ (by $71 \%$ in male rats and $59 \%$ in female rats, respectively). Given that COVID-19 prognosis is related to age and sex, ACE2 expression appears to be higher in young people than in elderly individuals and higher in females than in males and circulating ACE2 activity is increased in patients with cardiovascular complications [25]. The binding of SARS$\mathrm{CoV}-2$ spike protein to ACE2 has been shown to downregulate ACE2 and, in turn, to decrease angiotensin production. This mechanism may be involved in the pathogenesis of pulmonary hypertension and insufficiency caused by SARS-CoV-2 infection [26].

In a study on SARS-CoV-2 infection, Channappanavar et al. [27] showed that male mice were highly susceptible to this virus compared with age-matched female mice, confirming even in this infection, the protective function of ACE2. Estrogen, the main female sex hormone, is able to upregulate the expression of ACE2 [28] supporting the higher ACE2 expression in young women.

Another key discovery in understanding the mechanism of SARS-CoV-2 infection involves the role of the transmembrane serine protease 2 (TMPRSS2), a cell surface protein that is expressed by epithelial cells of specific tissues including those in the aerodigestive tract. Coronaviruses as well as influenza viruses critically depend on TMPRSS2 for viral entry and spread in the host. As a first step enabling host cell entry, the viral hemagglutinin protein attaches to ACE2 expressed on respiratory epithelial cells. In a second step, hemagglutinin is cleaved to activate internalization of the virus. This second step is dependent on the host cell, particularly TMPRSS2. Understanding how TMPRSS2 protein expression in the lung varies in women and men could reveal important insights into differential susceptibility to coronavirus infections. The strong regulation of TMPRSS2 by androgens have raised the hypothesis that the male predominance in the COVID-19 pandemic could partially be explained by TMPRSS2 $[29,30]$. Hence, also in this context, a link to genetic variations in the androgen receptor and COVID-19 disease severity has been suggested [31].

However, constitutive expression of TMPRSS2 in lung tissue does not appear to differ between men and women and low levels of androgens present in women may suffice to sustain TMPRSS2 expression [32]. In addition, TMPRSS2 expression levels may be also regulated by estrogen signaling [33].

Other biological factors may contribute to the disparity in sexspecific disease outcomes. In particular women, compared to men, have a better outcome of viral infections due to a stronger innate and acquired immune response. Sex-specific steroids play a role in this sex dimorphism in immune response with estrogens as immune enhancers and androgens as immune suppressors. Furthermore, the role played by X-linked genes (TLR-7, TLR8, CD40L, FOXP3 and CXCR3), which modulate the innate and adaptive immune response to virus infection and influence the immune response [34,35], is important.

The presence of two X chromosomes in women could lead to an over-expression of these X-linked immunoregulatory genes. As a consequence, women may have lower viral load levels than men, higher number of CD4+ T cells and higher levels of antibodies. In this context, the role played by the molecule Toll like receptor 7 (TLR7) [36] is very important.

The expression level of TLR7 in innate immune cells, is higher in women than in men leading to a higher immune response and viral infection resistance. TLR7 recognizes single strand RNA virus promoting the production of antibodies against the virus. Of note, in COVID-19 the presence of high levels of IL-6 and other inflammatory cytokines (the cytokine storm) is associated with a worse prognosis [37].

Interestingly, in women the production of inflammatory IL-6 after viral infection is lower than in males and is often correlated with a better longevity [38].

\section{Risk factors}

According to Italian data published on April $6^{\text {th }} 2020$ by the Istituto Superiore di Sanità (ISS), an overall prevalence of obesity of $10.0 \%$ was found among 1290 deceased patients, for whom medical records were available [39]; likewise, $72.1 \%$ of 775 patients in UK, with confirmed COVID-19 were overweight or obese and among patients with BMI $>30$ who had undergone intensive care, $60.9 \%$ of them died, according to the Intensive Care National Audit \& Research Centre (ICNARC) report on COVID19 in critical care of United Kingdom (27 March 2020) [40]. These data suggest that obesity may be a risk factor for severe COVID19 infection. Actually, obesity induces the immune dysregulation that appears, at least in part, to mediate the progression to critical illness and organ failure in a proportion of COVID-19 patients. In addition, increased levels of circulating pro-inflammatory cytokines, such as TNF- $\alpha$, IL- 6 , or C-reactive protein, have been reported in overweight and obese adult individuals. This may be a pro-inflammatory milieu that could be further exacerbated by COVID-19, possibly explaining the increased risk of severe outcome of COVID-19 in obese subjects. Given that overall more women are obese than men in developing countries, treatment of COVID-19 patients should be aware of the associated risk [41,42].

Smoking could be another risk factor of COVID-19 disease. It is related to higher expression of ACE2 [43]. The study conducted by Cai [44] did not find significant disparities in ACE2 gene expression between Asian and Caucasian groups, patients older and younger than 60 years, and men and women. This study observed significantly higher ACE2 gene expression in former smoker's lung compared to non-smoker's lung and found higher 
ACE2 gene expression in Asian current smokers compared to nonsmokers but not in Caucasian current smokers. In the studies conducted by Zhang [13] and by Guan [12] the percentage of current smokers' patients was low (1,4 and 12,6, respectively) and could not explain the association between smoking and the prevalence of SARS-CoV-2. However, Guan's study [12] there was an association between smoking and the severity of COVID-19 (11.8\% of patients with non-severe disease were smokers vs $16.9 \%$ with severe disease), but this data was not significative. For this reason other studies are needed to clarify the relation between smoking and prevalence or severity of COVID-19.

Hypovitaminosis D represents a risk factor for viral acute respiratory infection and COVID-19 [45]. In particular, vitamin D has three main mechanisms by which it reduces the risk of viral infection [46]. The first one consists in the physical barrier: vitamin D contributes to maintain junction integrity that could be broken by viruses. The second one regards the role of vitamin $\mathrm{D}$ in the enhancement of innate immunity and in the induction of antimicrobial peptide production, such as human cathelicidin, LL-3, and defensins [47]. Finally, vitamin D reduces the cytokine storm induced by the innate immune system, inhibiting the expression of pro-inflammatory cytokines and increasing the expression of antiinflammatory cytokines [48].

The inter-individual variance in vitamin $\mathrm{D}$ status could be involved in pulmonary inflammation and viral pathogenicity [49]. To note, serum vitamin D levels decrease with age, and this may be linked to the increased lethality of COVID-19 with age. Moreover, vitamin D levels were significantly reduced in both men and women with obesity. However, compared to women, serum vitamin $\mathrm{D}$ was consistently lower in men, irrespective of obesity and type 2 diabetes [50]. Hence, a link among vitamin D, gender, and COVID-risk should be considered and vitamin D supplements could be a valid adjuvant for prevention of this severe infection, in particular in elderly men [51].

\section{Clinical presentation}

In a multicenter European study [52] including 417 patients, the mean age was $36.9 \pm 11.4$ years (range 19-77) and 263 females and 154 males were enrolled: European (93.3\%), South American (2.7\%), Sub-Saharan African (2.2\%), Black African (1.4\%), Asian $(0.2 \%)$, and North American (0.2\%). The females were proportionally more affected by hyposmia or anosmia compared to males $(p<0.001)$. Similar results were found for gustatory dysfunction $(p=0.001)$. There are no gender-related data of eyes' involvement.

The retrospective study of Mo [53] included 155 patients with confirmed COVID-19 and showed that the patients with "refractory" COVID-19 were often male with manifestations of anorexia and fever on admission. Those patients received oxygen, expectorant and adjunctive agents $(p<0.05)$ when considering the factors of disease severity on admission, mechanical ventilation, and ICU transfer.

\section{Comorbidities}

In the study conducted by Grasselli in Lombardy (Nothern Italy) [16] hypertension was the most common comorbidity (49\%), followed by the cardiovascular disease (21\%), hypercholesterolemia (18\%) and diabetes $(17 \%)$. Those data were similar to two Chinese studies $[4,12]$ in which a history of chronic obstruc- tive pulmonary disease was only reported in $4 \%$ of the patients, i.e. more hypertensive, significantly older and died in the intensive care unit (ICU) patients.

A review conducted by Cheng [21] underlined that the poor prognosis of patients with COVID-19 was related to factors such as male sex, age $>60$ years, underlying diseases (hypertension, diabetes and cardiovascular disease) and complicating secondary acute respiratory distress syndrome (ARDS). Although COVID-19 is predominantly a respiratory illness, a large number of COVID-19 patients exhibit severe cardiovascular damage and those with preexisting CVD appear to have an increased risk of death.

Individuals with diabetes mellitus had also a significantly increased risk of hospitalization and death in the pandemic COVID-19. It was not fully understood if diabetes constitutes an increased risk of infection but it was known that individuals with diabetes had a dysregulated immune system and were more susceptible to infections. Moreover, type 2 diabetes had been associated with reduced ACE2 activity in late diabetes [54].

The sex differences in clinical outcomes could also be cause by pre-existing comorbidities, such as hypertension, cardiovascular disease and diabetes, which were almost always present in severe and lethal COVID-19 cases and tended to be more frequent and more severe in men [55].

\section{Diagnosis}

In the study conducted by Liu [56], 4880 cases (2251 male and 2629 female) with respiratory infection symptoms or close contact with COVID-19 patients in hospital in Wuhan were tested for SARS-CoV-2 infection by the use of quantitative RT-PCR (qRTPCR) on samples from the respiratory tract. The positive rate was significantly higher in men $(40.43 \%)$ than in women $(36.71 \%)$ and increased from $24.9 \%$ (age $18-30$ ) to $61.81 \%$ (age $>70$ ), confirming that male patients and older population had significant higher positive rates.

\section{Prognosis and mortality}

Based on the available data, older male adults and people of any age who have chronic underlying morbidities might be at higher risk for severe illness and fatal outcome from COVID-19.

The meta-analysis conducted by $\mathrm{Li}$ [11] suggested that the discharge rate of patients with COVID-19 was $52 \%$, with a fatality rate of $5 \% .31 .5 \%$ of the deceased patients were frail and had one or more of the following cases: advanced age ( $>60$ years), cancer, more underlying diseases, or major infections.

The retrospective study conducted by Zhou et al. [10] showed that the female fatality rate was $30 \%$, while that of the males was $70 \%$ and confirmed that death, in patients with COVID-19, was associated with increased age.

The retrospective study conducted by Chen [57] recruited 799 inpatients of whom 113 died, with a mortality rate of up to $14.1 \%$, and 161 were discharged. The median age of deceased patients (68 years) was significantly older than total recovered patients (51 years). The male sex was more predominant in deceased patients $(83 ; 73 \%)$ than in recovered patients $(88 ; 55 \%)$. Chronic hypertension $(48 \%)$ and other cardiovascular comorbidities (14\%) were more frequent among deceased patients than recovered patients (24\% and $4 \%$, respectively). 
The prospective study conducted on COVID-19 pneumonia by $\mathrm{Du}$ [58] included a total of 179 patients $(54,2 \% \mathrm{M}, 45,8 \% \mathrm{~F})$ with a mean age that was 57.6 years. Survivors were 158 (88.8\%) of whom male $(55.1 \%)$ and female $(44.9 \%)$. Mortality in male patients was $47.6 \%$ and in female patients $52.4 \%$. The patients in the deceased group were much older than those in survivor group (70.2 \pm 7.7 years versus $56.0 \pm 13.5$ years, $\mathrm{p}<0.001)$. The authors identified four risk factors: age $\geq 65$ years, preexisting concurrent cardiovascular or cerebrovascular diseases, $\mathrm{CD}^{+} \mathrm{CD}^{+} \mathrm{T}$ cells $\leq 75$ cell $\mu \mathrm{l}^{-1}$, and cardiac troponin $\mathrm{I} \geq 0.05 \mathrm{ng} \cdot \mathrm{ml}^{-1}$; the latter two factors were better predictors for mortality in COVID-19 pneumonia patients.

Dudley et al. [59] showed in China a peak morbidity in the 5059 years cohort, while in the Republic of Korea in the 20-29 years cohort. In the systematic review of Fu et al. [60] the overall estimated proportion of severe cases and case-fatality rate was $25.6 \%$ and $3.6 \%$ respectively. The case fatality rate and laboratory abnormalities were higher in severe cases, in patients from Wuhan, and in older patients, but did not differ by sex. The report of the WHOChina Joint Mission on COVID-19 [14] confirmed that the mortality rate was greater in males $(4.7 \%)$ than in females $(2.8 \%)$. The Korean Report [15] showed that the case fatality rate increased with older age. The case fatality rate of persons 50 years or older was higher than that of persons younger than 50 years $(1.2 \% \mathrm{vs}$ $0.2 \%, \mathrm{p}=0.0017)$. There were no sex differences.

In Italy, deaths are similar to China with fatalities mostly in the elderly with known comorbidities [61]. According to the ISS report [62] based on available data on May $7^{\text {th }}$, the mean age of patients dying for COVID-19 was 80 years (median age 81 years) and women were $39.1 \%$. Deceased women had an older median age than men (85 vs 79 years). There were no sex differences about the most common pre-existing chronic pathologies in deceased patients.

\section{Conclusions}

In our review we underlined that in China men were more infected by COVID-19 than women. Most patients were aged 3079 years, and men with underlying diseases, including diabetes, hypertension and cardiovascular disease developed severe condition with an increased mortality rate. Instead in Korean outbreak, two peaks of age groups were described: 20 years and 50 years, with a predominance in the age group of the 20-year-olds. Predominance in the age group of the 20 year-olds and females may be linked to the outbreak in a religious group in Daegu; moreover, the case fatality rate of persons 50 years or older was higher than that of persons younger than 50 years.

In Italy, in particular in Lombardy region, men were more infected by COVID-19 than women, the median age was 63 years and deaths are similar to China with deaths mostly in the elderly with known comorbidities. Many factors can contribute to disparity in sex-specific disease outcomes such as sex-specific steroids and activity of X-linked genes which modulate the innate and adaptive immune response to virus infection and influence the immune response. Moreover, the male predominance in the COVID-19 pandemic could partially be explained by TMPRSS2. The females were proportionally more affected by hyposmia or anosmia and gustatory dysfunction compared with males. There were not available data about eyes' involvement. The sex differences in clinical outcomes could be also caused by pre-existing comorbidities, such as hypertension, cardiovascular disease and diabetes, which were almost always present in severe and lethal COVID-19 cases and tended to be more frequent and more severe in men.
Because a specific treatment is not yet available, more clinical and basic research regarding gender and other prognostic factors for individualized risk assessment and management strategies are crucial. Currently, there is only data available on the sex indicator for admitted or deceased patients. There is no data about domestic isolation and there is no analysis about other gender indicators.

The data considered in our study are the only currently available in the literature, but it is appropriate to implement a specific analysis with all gender indicators to identify appropriate strategies. Moreover, the evaluation of the health service efficacy is a key element in determining gender outcomes.

In our opinion, it is very important to carry out a gender analysis of data about COVID-19 as well as other specific studies to evaluate gender differences. Knowing the gender differences in COVID-19 outbreak would be a fundamental tool to understand the effects of a health emergency on individuals and communities as well as to carry out effective and equitable policies, public health measures and targeted solutions.

\section{References}

1. WHO. Clinical management of severe acute respiratory infection (SARI) when COVID-19 disease is suspected. https://www.who.int/publications-detail/clinical-managementof-severe-acute-respiratory-infection-when-novel-coronavirus-(ncov)-infection-is-suspected

2. WHO. WHO Coronavirus Disease (COVID-19) Dashboard Available from: https://who.sprinklr.com/

3. Ministero della Salute. Nuovo coronavirus. Available from: http://www.salute.gov.it/nuovocoronavirus

4. Wang D, Hu B, Hu C, et al. Clinical characteristics of 138 hospitalized patients with 2019 novel coronavirus-infected pneumonia in Wuhan, China. JAMA 2020;323:1061.

5. Wu Z, McGoogan JM. Characteristics of and important lessons from the Coronavirus Disease 2019 (COVID-19) outbreak in China: summary of a report of 72314 cases from the Chinese Center for Disease Control and Prevention. JAMA 2020. doi: 10.1001/jama.2020.2648.

6. Epidemiology Working Group for NCIP Epidemic Response, Chinese Center for Disease Control and Prevention. [The epidemiological characteristics of an outbreak of 2019 novel coronavirus diseases (COVID-19) in China].[Article in Chinese with English abstract]. Zhonghua Liu Xing Bing Xue Za Zhi 2020;41:145-51. doi: 10.3760/cma.j.issn.0254-6450. 2020.02.003.

7. Wang Z, Yang B, Li Q, et al. Clinical features of 69 cases with Coronavirus disease 2019 in Wuhan, China. Clin Infect Dis. 2020.

8. Huang C, Wang Y, LI X, et al. Clinical features of patients infected with 2019 novel coronavirus in Wuhan, China. Lancet 2020;395:497-506.

9. Chen N, Zhou M, Dong X, et al. Epidemiological and clinical characteristics of 99 cases of 2019 novel coronavirus pneumonia in Wuhan, China: a descriptive study. Lancet 2020;395: 507-13.

10. Zhou F, Yu T, Du R, et al. Clinical course and risk factors for mortality of adult inpatients with COVID-19 in Wuhan, China: a retrospective cohort study. Lancet 2020;395(10229):10541062.

11. Li LQ, Huang T, Wang YQ, et al. 2019 novel coronavirus patients clinical characteristics, discharge rate and fatality rate 
of meta- analysis. J Med Virol 2020;10.1002/jmv.25757. doi: 10.1002/jmv. 25757.

12. Guan W, Ni Z, Liang W. Clinical characteristics of coronavirus disease 2019 in China. N Engl J Med 2020;382:1708-20. doi: 10.1056/NEJMoa2002032.

13. Zhang JJ, Dong X, Cao Y. Clinical characteristics of 140 patients infected with SARS-CoV-2 in Wuhan, China. Allergy 2020. doi: 10.1111/all.14238.

14. WHO. Report of the WHO-China Joint Mission on Coronavirus Disease 2019 (COVID-19). Available from: https://www.who.int/publications-detail/report-of-the-whochina-joint-mission-on-coronavirus-disease-2019-(covid-19)

15. Korean Society of Infectious Diseases, Korean Society of Pediatric Infectious Diseases, Korean Society of Epidemiology, et al. Report on the Epidemiological Features of Coronavirus Disease 2019 (COVID-19) Outbreak in the Republic of Korea from January 19 to March 2, 2020. J Korean Med Sci 2020;35:e112. doi: 10.3346/jkms.2020.35.e112.

16. Grasselli G, Zangrillo A, Zanella A, et al. Baseline characteristics and outcomes of 1591 patients infected with SARS-CoV-2 admitted to ICUs of the Lombardy Region, Italy. JAMA 2020;323:1574-81. doi: 10.1001/jama.2020.5394.

17. Lovato A, de Filippis C. Clinical presentation of COVID-19: A systematic review focusing on upper airway symptoms. Ear Nose Throat J 2020;145561320920762. doi: 10.1177/ 0145561320920762 .

18. ISTAT. Impatto dell'epidemia COVID-19 sulla mortalità totale della popolazione residente primo trimestre 2020. Rapporto ISTAR-ISS 4 maggio 2020. Available from: https://www. istat.it/it/files//2020/05/Rapporto_Istat_ISS.pdf

19. Chu J, Yang N, Wei Y, et al. Clinical characteristics of 54 medical staff with COVID-19: A retrospective study in a single center in Wuhan, China. J Med Virol 2020:10.1002/jmv.25793. doi: $10.1002 /$ jmv. 25793

20. Zhu A, Zhang DZ, Wang W, et al. A novel coronavirus from patients with pneumonia in China, 2019. N Engl J Med 2020; 382:727-33.

21. Cheng H, Wang Y, Wang GQ. Organ-protective effect of angiotensin-converting enzyme 2 and its effect on the prognosis of COVID-19. J Med Virol 2020. doi: 10.1002/jmv.25785.

22. Zhou P, Yang X-L, Wang X-G, et al. A pneumonia outbreak associated with a new coronavirus of probable bat origin. Nature 2020;579:270-3. doi: 10.1038/s41586-020-2012-7.

23. Zhao Y, Zhao Z, Wang Y, at al. Single-cell RNA expression profiling of ACE2, the putative receptor of Wuhan 2019-nCov. bioRxiv 2020. Available from: https://www.biorxiv.org/content/10.1101/2020.01.26.919985v1

24. Xie X, Xudong X, Chen J, et al. Age- and gender-related difference of ACE2 expression in rat lung. Life Sciences 2006;78:2166-71.

25. Soro-Paavonen A, Gordin D, Forsblom C, et al. Circulating ACE2 activity is increased in patients with type 1 diabetes and vascular complications. J Hypertens 2012;30:375-83.

26. Gurwitz D. Angiotensin receptor blockers as tentative SARSCoV-2 therapeutics. Drug Dev Res 2020. doi: 10.1002/ddr. 21656.

27. Channappanavar R, Fett C, Mack M, et al. Sex- based differences in susceptibility to SaRs_ Cov infection I. Immunol 2017;198:4046-53.

28. Bukowska A, Spiller L, Wolke C, et al. Protective regulation of theACE2/ACE gene expression by estrogen in human atrial tissue from elderly men. Exp Biol Med (Maywood) 2017;242: 141223 .
29. Wambier CG, Goren A. SARS-COV-2 infection is likely to be androgen mediated. J Am Acad Dermatol 2020. pii: S01909622(20)30608-3.

30. Sharifi N, Ryan CJ. Androgen hazards with COVID-19. Endocr Relat Cancer 2020;27:E1-3.

31. McCoy J, Wambier CG, Vano-Galvan S, et al. Racial variations in COVID-19 deaths may be due to androgen receptor genetic variants associated with prostate cancer and androgenetic alopecia. Are anti-androgens a potential treatment for COVID-19? J Cosmet Dermatol 2020. doi: 10.1111/jocd. 13455.

32. Stopsack KH, Mucci LA, Antonarakis ES, et al. TMPRSS2 and COVID-19: Serendipity or opportunity for intervention? Cancer Discov 2020. doi: 10.1158/2159-8290.CD-20-0451.

33. Setlur SR, Mertz KD, Hoshida Y, et al. Estrogen-dependent signaling in a molecularly distinct subclass of aggressive prostate cancer. J Natl Cancer Inst 2008;100:815-25.

34. Klein SL, Flanagan KL. Sex differences in immune responses. Nat Rev Immunol 2016;16:626-38.

35. Bouman A, Heineman, MJ, Faas MM. Sex hormones and the immune response in humans. Hum Reprod 2005;11:411-23.

36. de Groot NG, Bontrop RE. COVID-19 pandemic: is a genderdefined dosage effect responsible for the high mortality rate among males? Immunogenetics 2020. doi: 10.1007/s00251020-01165-7.

37. Hirano T, Murakami M. COVID-19: A New virus, but a familiar receptor and cytokine release syndrome. Immunity 2020 . pii: S1074-7613(20)30161-8.

38. Zhu Z, Cai T, Fan L, et al. Clinical value of immune-inflammatory parameters to assess the severity of coronavirus disease 2019. Int J Infect Dis 2020;95:332-339. doi: 10.1016/j.ijid. 2020.04.041.

39. Epicentro ISS [Internet]. Characteristics of COVID-19 patients dying in Italy. Accessed on: 7 April 2020. Available from: https://www.epicentro.iss.it/en/coronavirus/sars-cov-2-analysis-of-deaths.

40. ICNARC [Internet]. Accessed on: 3 April 2020. Available from: www.icnarc.org.

41. Kanter R, Caballero B. Global gender disparities in obesity: A review. Adv Nutr 2012;3:491-8.

42. Muscogiuri G, Pugliese G, Barrea L, et al. Obesity: The "Achilles heel" for COVID-19? Metabolism 2020;108: 154251. doi: 10.1016/j.metabol.2020.154251.

43. Cai H. Sex difference and smoking predisposition in patients with COVID-19. Lancet Respir Med 2020;8:e20. doi: 10.1016/S2213-2600(20)30117-X

44. Cai G. Bulk and single-cell transcriptomics identify tobacco-use disparity in lung gene expression of ACE2, the receptor of 2019nCov. medRxiv 2020. Available from: https:/www.medrxiv.org/ content/10.1101/2020.02.05.20020107v3.

45. Grant WB, Lahore H, McDonnell SL, et al. Evidence that vitamin D supplementation could reduce risk of influenza and COVID-19 infections and deaths. Nutrients 2020;12:e988.

46. Schwalfenberg GK. A review of the critical role of vitamin D in the functioning of the immune system and the clinical implications of vitamin D deficiency. Mol Nutr Food Res 2011;55:96-108.

47. Laaksi I. Vitamin D and respiratory infection in adults. Proc Nutr Soc 2012;71:90-97.

48. Silberstein M. Vitamin D: A simpler alternative to tocilizumab for trial in COVID-19? Med Hypotheses 2020;140: 109767

49. Carter SJ, Baranauskas MN, Fly AD. Considerations for obe- 
sity, vitamin D, and physical activity amidst the COVID-19 pandemic. Obesity 2020. doi: 10.1002/oby.22838.

50. Sanghera DK, Sapkota BR, Aston CE, Blackett PR. Vitamin D status, gender differences, and cardiometabolic health disparities. Ann Nutr Metab 2017;70:79-87.

51. La Vignera S, Cannarella R, Condorelli RA, et al. Sex-specific SARS-CoV-2 mortality: Among hormone-modulated ACE2 expression, risk of venous thromboembolism and hypovitaminosis D. Int J Mol Sci 2020;21:e2948.

52. Lechien JR, Chiesa-Estomba CM, De Siati DR, et al. Olfactory and gustatory dysfunctions as a clinical presentation of mildto-moderate forms of the coronavirus disease (COVID-19): a multicenter European study. Eur Arch Otorhinolaryngol 2020. doi: 10.1007/s00405-020-05965-1.

53. Mo P, Xing Y, Xiao Y et al. Clinical characteristics of refractory COVID-19 pneumonia in Wuhan, China. Clin Infect Dis 2020. doi: 10.1093/cid/ciaa270.

54. Angelidi AM, Belanger MJ, Mantzoros CS. COVID-19 and diabetes mellitus: what we know, how our patients should be treated now, and what should happen next. Metabolism 2020. doi: 10.1016/j.metabol.2020.154245.

55. Zheng Z, Peng F, Xu B, et al. Risk factors of critical \& mortal COVID-19 cases: A systematic literature review and metaanalysis. J Infect 2020. doi: 10.1016/j.jinf.2020.04.021

56. Liu R, Han H, Liu F, et al. Positive rate of RT-PCR detection of SARS-CoV-2 infection in 4880 cases from one hospital in Wuhan, China, from Jan to Feb 2020. Clin Chim Acta 2020;505:172-5.

57. Chen T, Wu D, Chen H, et al. Clinical characteristics of 113 deceased patients with coronavirus disease 2019: retrospective study. BMJ 2020;368:m1091. Erratum in BMJ 2020; 368:m1295.

58. Du RH, Liang LR, Yang CQ, et al. Predictors of mortality for patients with COVID-19 pneumonia caused by SARS-CoV-2: A prospective cohort study. Eur Respir J 2020. doi: 10.1183/13993003.00524-2020.

59. Dudley JP, Lee NT. Disparities in age-specific morbidity and mortality from SARS-CoV-2 in China and the Republic of Korea. Clin Infect Dis 2020. doi: 10.1093/cid/ciaa354.

60. Fu L, Wang B, Yuan T, et al. Clinical characteristics of coronavirus disease 2019 (COVID-19) in China: a systematic review and meta-analysis. J Infect 2020;80:656-65. doi: 10.1016/j. jinf.2020.03.041.

61. Porcheddu R, Serra C, Kelvin D, et al. Similarity in case fatality rates (CFR) of COVID-19/SARS-COV-2 in Italy and China. J Infect Dev Ctries 2020;14:125-8. doi: 10.3855/jidc.12600.

62. Epicentro ISS. Characteristics of SARS-CoV-2 patients dying in Italy Report based on available data on May 7th, 2020. Available from: https://www.epicentro.iss.it/en/coronavirus/bollettino/ Report-COVID-2019_7_May_2020.pdf 